\begin{abstract}
Grief is a normal and natural response to the loss of a loved one that is characterized by a broad range of emotional, behavioral, cognitive, and physiological symptoms. For most bereaved, these symptoms abate over time as the individual comes to terms with the loss. However, about $10 \%$ of the bereaved experience a persistent and debilitating grief reaction, a condition that has been termed complicated grief or prolonged grief. Self-compassion has been defined as a kind and caring attitude towards oneself when experiencing suffering. The present study explored the associations between self-compassion and complicated grief symptoms in a bereaved sample from the general population. Participants completed measures of complicated grief (Inventory of Complicated Grief), self-compassion (Self-Compassion Scale), and neuroticism (NEO FFI-3 neuroticism scale). In line with previous studies, bereavement by unexpected death and high neuroticism were related to grief symptom severity. The results further showed statistically significant relationships between low self-compassion and the severity of complicated grief symptoms. No associations between the cause of death (natural expected, natural sudden, or unnatural) and self-compassion were found. The self-kindness component of self-compassion did not moderate the relationships between established risk factors for complicated grief (cause of death and neuroticism) and complicated grief symptomatology. It is concluded that it is important for clinicians to be attentive to the role that low self-compassion may have in the grief response of bereaved individuals.
\end{abstract}

Keywords: Bereavement, complicated grief, self-compassion; neuroticism 


\section{Associations between self-compassion and complicated grief symptoms in bereaved individuals: An exploratory study}

Grief is a normal response when an important relationship is permanently broken as a result of death and characterized by a variety of emotional, behavioral, cognitive, and physiological symptoms, including sadness, longing, social withdrawal, and sleep problems (Shear, 2012; Shear, Ghesquiere, \& Glickman, 2013; Worden, 2009). Early attempts to understand and systematize responses to loss regarded the grief process as a series of subsequent stages in which the bereaved processed various aspects of the loss (e.g., Kübler-Ross, 2009). However, this type of grief model has shown to be too rigid compared to the many ways grief is experienced and the waves of emotions seen in grieving individuals over time (Dyregrov, 2006; Wortman \& Boerner, 2011). Recent models of grief are more oriented towards the interaction between cognition and emotions in bereavement and consider severe and prolonged grief reactions to be the result of a negative interaction between these factors (e.g., Boelen, van den Hout, \& van den Bout, 2006; Stroebe \& Schut, 2010). A relatively new contribution to grief research has been the so-called dual process model of coping with bereavement (Stroebe \& Schut, 1999, 2010) that considers grief as consisting of the parallel processes of orientation towards emotional processing of the loss and the future-oriented restoration of the bereaved' $\mathrm{s}$ life. The purpose of the present study was to explore the associations between self-compassion, defined as a kind and open attitude towards one's own suffering and failures (Neff, 2003), and the severity of grief symptoms.

Most bereaved come to terms with the loss gradually, accepting and integrating its reality into life. However, for a considerable number of bereaved, the tasks of loss-oriented and restoration-oriented coping poses a profound challenge, resulting in persisting and intense grief 
reactions that are highly distressing and impair psychosocial functioning. This condition has been labeled complicated grief (CG; Shear et al., 2011) or prolonged grief (Prigerson et al., 2009). It is estimated that approximately $10 \%$ of bereaved individuals suffer from complicated grief (Lundorff, Holmgren, Zachariae, Farver-Vestergaard, \& O’Connor, 2017). In addition to being a severe mental health problem, CG has also shown to be associated to physical health problems, e.g., higher risk of cardiovascular disease and generally increased mortality (Stroebe, Schut, \& Stroebe, 2007). A recent systematic review identified, among other factors (e.g., the type and quality of the relationship to the deceased, low social support), the circumstances of the death and the personality trait of neuroticism as strong risk factors for the development of CG (Burke \& Neimeyer, 2013; see also Piper, Ogrodniczuk, Joyce, \& Weideman, 2011). Unexpected deaths are associated with greater loss of function and longer grief reactions (Kristensen, Weisæth, \& Heir, 2012), which may be due to the lack of time to prepare for the death. Unexpected deaths are also more often linked to traumatic circumstances such as killing, natural disasters, or traffic accidents. Media coverage of the traumatic incident can increase the burden on the bereaved (Kristensen et al., 2012; Kristensen, Weisæth, Hussain, \& Heir, 2015). Moreover, violent death is related to PTSD symptoms and the persistence of depression over time (Kaltman \& Bonanno, 2003), which can affect the severity and course of the grief response. The personality trait of neuroticism has consistently shown associations with severe grief reactions (e.g., Bonanno et al., 2002; Wijngaards-de Meij et al., 2007). Neuroticism can be described as the tendency to respond strongly with negative emotions to stress, e.g., sadness, anxiety, or anger (Barlow, Sauer-Zavala, Carl, Bullis, \& Ellard, 2014).

Self-compassion (SC) is a relatively young concept in Western psychology that was introduced and defined by Kristin Neff (2003b). In her approach, Neff (2003b) used Buddhist 
teaching about compassion as a starting point and proposed that compassion is about seeing pain and reacting with tenderness and care to the sufferer. According to (Neff, 2003b), SC is about expanding this goodness to include oneself, i.e., the ability to recognize one's own pain and meet it with warmth, care, and a desire to relieve it just as one would meet a good friend or loved family member in the same situation (Neff \& Vonk, 2009). SC can contribute to increased psychological and somatic well-being by enabling the individual to relieve his or her own suffering and providing oneself the warmth one needs to cope with challenges in life (Neff, 2003b). In Neff's (2003b) model, SC has three components, each comprising a positive and a negative dimension. The first component of SC is self-kindness versus self-judgment. Selfkindness refers to a gracious attitude to one's own imperfection and the ability to engage in a friendly inner dialogue with oneself in the face of adversity (Neff, 2003b). Highly self-critical people, on the other hand, often show the opposite pattern of inner dialogue, characterized by repetitive demoralizing and destructive thoughts that gives rise to several negative effects, such as low self-esteem or lack of impetus for initiating new projects due to fear of failure (Gilbert, 2009; Neff, 2003b). Common humanity is the second component of SC. Common humanity involves the idea that the experience of failures and suffering is an inevitable part of being human and a shared experience of all human beings (Neff, 2003b). The negative dimension of common humanity is isolation. People who score high on isolation tend to regard their failures and limitations as something separating them from their fellow human beings and as something that makes them less worthwhile (Neff, 2003b). The third component of SC is mindfulness, which is defined as an attentive and non-judgmental attitude towards one's experiences. Mindfulness is about being aware of one's feelings and thoughts, but observing them without being absorbed in them (Bishop et al., 2004; Neff, 2003b). Accordingly, the negative dimension 
of mindfulness is over-identification with emotions (Shapiro \& Carlson, 2009), which implies that the individual does not distinguish between the present and transient emotional state and her or his core self. Over-identification is associated with rumination and prolongation of negative affects (Rude, Little Maestas, \& Neff, 2007). According to Neff (2003b), the three components of SC are not understood as separate concepts, but rather as mutually dependent, reinforcing, and interrelated parts of an attitude towards oneself. For example, the ability to show self-kindness will depend on being mindful towards one's feelings. Similarly, attention to the feeling of being human can make the individual more aware of how the current experience is something that all people share as a whole. Understanding of our general shortcomings as human beings can motivate the individual show more kindness to oneself.

An increasing body of research shows associations between low SC and a variety of psychological disorders, including depression and anxiety (MacBeth \& Gumley, 2012), eating disorders (Braun, Park, \& Gorin, 2016), and psychosis (Eicher, Davis, \& Lysaker, 2013). The negative dimensions of the three components of SC show consistently stronger relationships with psychopathology than the positive dimensions (Muris \& Petrocchi, 2016). Self-compassion is also strongly negatively associated with neuroticism (Neff, Rude, \& Kirkpatrick, 2007; Pfattheicher, Geiger, Hartung, Weiss, \& Schindler, 2017). Composites of the positive components of SC have been found to moderate the relationships between self-coldness and depressive symptoms (Dundas, Svendsen, Wiker, Granli, \& Schanche, 2016; Körner et al., 2015). Further, findings suggest that SC buffers the effect of low self-esteem on mental health in adolescents (Marshall et al., 2015).

Understanding factors that contribute to CG symptomatology is important for the development of new interventions or refinement of existing treatments. Although it has been 
suggested that SC is beneficial for individuals grieving a loss (Shear, 2014), an empirical investigation of the associations between SC and symptoms of CG has not yet been undertaken. Thus, the purpose of the present study was to explore the relationships between the three components of SC proposed by Neff (2003b) and symptoms of CG in a bereaved sample. A second goal of the current investigation was to investigate if the positive dimensions of SC moderates the relationships between the risk factors cause of death and neuroticism and symptoms of CG.

\section{Method}

\section{Participants}

Data were collected between August and November 2016. Participants were recruited via Facebook and by email via a local educational institution. The email was sent to the administration of the institution, which forwarded it to its employees. The Facebook message and the email contained an invitation to participate in a research study about self-care and grief reactions after personal losses. Anonymous and voluntary participation was emphasized, and it was indicated that the survey takes approximately 10-15 minutes to complete. Sharing of the study invitation was encouraged. The Facebook message and the email invitation included a link to an online survey tool. The information about the study along with contact information for the researchers was reiterated at the beginning of the survey. As no personally identifiable information was obtained, approval from the Regional Committee for Medical and Health Research was not required. The criterion for starting the survey was agreeing to an informed consent statement. In addition to age and gender of the respondent, information about the deceased's relation to the participant was obtained, as well as the age of the deceased at the time 
of death and the type of death. One hundred and fifty-eight individuals responded to the survey. Six participants were excluded from the current study because they reported answering the questions based on the loss of a pet (2) or multiple losses (4). The average age of the participants in the final sample was 43.3 years $(\mathrm{SD}=15.6$ years). The sample consisted of $119(78.3 \%)$ women and $33(21.7 \%)$ men. On average, it was 9.8 years since the death (with a SD of 11.2 years, the information given by 22 participants was ambiguous, i.e., entered only as a number without indicating the unit of time, and registered as missing). The mean age of the deceased was 58.6 years $(\mathrm{SD}=24.5$, one data point missing). Regarding the relationship with the deceased, most participants reported grieving the loss of a parent $(n=49,32.2 \%)$, followed by a grandparent $(n=41,27 \%)$, a friend $(n=22,14.5 \%)$, another relative $(n=12,7.9 \%)$, a partner $(n$ $=11,7.2 \%)$, a sibling $(n=9,5.9 \%)$, a child $(n=4,2.6 \%)$, other $(n=3,2 \%)$, and colleague $(n=$ 1, 1\%). Cause of death was reported as natural expected (e.g., after prolonged illness, old age, congenital defects, or the like) by $46.7 \%(n=71)$, as natural sudden (e.g., heart attack, stroke, short or acute illness, undiagnosed illness, or the like) by $32.9 \%(n=50)$, as unnatural sudden (e.g., accident, poisoning, overdose, or assault) by $14.5 \%(n=22)$, and as suicide by $5.9 \%(n=$ 9) of the participants.

\section{Measures}

Inventory of Complicated Grief (ICG; Prigerson et al., 1995).

In order to assess symptoms of CG, the Norwegian translation of the ICG (Prigerson et al., 1995) was included in the study. The ICG is a self-report inventory consisting of 19 items. The ICG captures symptoms of CG reactions, such as shock and unrealness, longing, and anger over the loss. The response options indicate the severity of the symptoms on a 5-point scale from 
0 (never) to 4 (always). The clinical cut-off is a score of 25 or larger (Prigerson et al., 1995). In the present study, the scale had a reliability of .92 (omega total).

\section{Self-Compassion Scale (SCS; Neff, 2003a)}

To assess SC, the Norwegian version of the SCS (Neff, 2003a) was administered. The SCS consists of 26 items that measure the three positive and the three negative dimensions of SC. Items are answered along a 5-point scale ranging from 1 (almost never) to 5 (almost always). The three positive and the three negative subscales are summarized to self-compassion and selfcoldness composites, respectively. The appropriateness of computing a SCS total score is currently under debate (Muris, Otgaar, \& Petrocchi, 2016; Neff, 2016). As the majority of findings favor a two-factor solution (self-compassion vs self-coldness) (e.g., Brenner, Heath, Vogel, \& Credé, 2017; Costa, Marôco, Pinto-Gouveia, Ferreira, \& Castilho, 2016; López et al., 2015; Pfattheicher et al., 2017) (see however Neff, Whittaker, \& Karl, 2017), the SCS total score was not used in the present study. The SCS-dimensions had the following reliabilities (omega total) in the current sample: self-kindness .88 , self-judgment .86 , common humanity .91 , isolation .90 , mindfulness .82 , and over-identified .88 . The positive and negative composites had omega totals of .92 and .94 , respectively.

\section{Neuroticism scale of the NEO- Five-Factor Inventory-3 (NEO-FFI-3; McCrae \&}

Costa, 2010)

Neuroticism was assessed with the neuroticism scale of the NEO-FFI-3 (McCrae \& Costa, 2010). The NEO-FFI-3 neuroticism scale consists of 12 items that are answered on a fivepoint scale from 0 (strongly disagree) to 4 (strongly agree). In the present sample, the scale had an omega total of .91 .

\section{Analyses}


Omega total was used to estimate the reliability of the measures included in the present study. Omega total was preferred to Cronbach's alpha as omega total is considered a better index of reliability (e.g., Peters, 2014). The formula for omega total is similar to the formula for computing Cronbach's alpha but omega total does not require tau equivalence (McNeish, 2018). Descriptive statistics for the sample were calculated. One-way ANOVAs with LSD post hoc tests were used to investigate the associations between categorical variables and complicated grief symptoms and the six components of SC. When Levene's test indicated that the assumption of homogeneity of variances was violated, the Welch F-statistic was calculated, and Games-Howell post hoc statistics were used. Bivariate correlations between were obtained to examine the associations between continuous variables. $95 \%$ confidence intervals for the correlations were estimated using 1000 bootstrap samples. A hierarchical linear regression was conducted to examine the prediction of complicated grief symptoms from the SC dimensions when controlled for characteristics of the sample. In the first step, variables related to the deceased and the death (i.e., relationship to the deceased, time since the death, age of the deceased, and cause of death) and the participants (age and gender) were included. Categorical variables (relationship to the deceased and cause of death) were dummy coded before they were entered into the regression. In the second step, the six scales of the SCS were entered simultaneously. Moderation of the positive SC composite between the risk factors neuroticism and cause of death and complicated grief symptoms were tested in two regression analyses, in which the ICG total score was predicted by the risk factor, the positive SC composite, and the interaction between the two variables. Moderation was assumed to occur when the interaction between the risk factor and self-compassion was significant at $p<.05$. 
The statistical analyses were conducted in the psych package 1.7.5 for R (Revelle, 2017) (omega total, confidence intervals for correlations) and SPSS 24 (descriptives, correlations,regression analyses, ANOVAs). Process 3.0 (Hayes, 2017) was used for the moderation analyses in SPSS.

\section{Results}

The average score of the ICG in the sample was $16.8(\mathrm{SD}=10.9)$. Thirty-four participants $(22.4 \%)$ had ICG-scores of 25 or above. Grief severity was related to cause of death $(F=6,53,3,148, p<.001)$, the relationship with the deceased $(F=10,63,7,24,31, p<.001)$, and the age of the deceased $(r=-.38,95 \%$ CI: -.51 to $-.25, p<.001)$. Post hoc tests showed that individuals bereaved by unexpected deaths had higher scores on the ICG (unnatural sudden: $M=$ $20.41, S D=12.25$, natural sudden: $M=20.06, S D=11.15$, suicide: $M=21.00 ; S D=7.89$ ) than those bereaved by expected deaths $(M=12.83 ; S D=9.32)($ all $p \mathrm{~s}<.05)$. Individuals grieving the loss of a grandparent $(M=9.76, S D=7.01)$ had lower scores on the ICG than individuals grieving the loss of child $(M=24.00, S D=2.83)$, sibling $(M=24.00, S D=6.58)$, or parent $(M=$ $17.16, S D=10.82)$. The ICG scores of individuals who had lost a partner $(M=22.09, S D=$ 8.63), other relative $(M=24.25, S D=14.52)$, or a friend $(M=18.64, S D=11.88)$ did not differ significantly from the other groups. There were no significant associations between the ICG scores and the participants' age $(r=-.06,95 \% \mathrm{CI}:-.22$ to $.11, p=.470)$ and gender (female: $M=$ 17.66, $S D=11.21$; male: $M=13.67, S D=9.15 ; F=3,52,1,151, p=.063)$, and the time since the death $(r=-.16,95 \%$ CI: -.30 to $.00, p=.065)$. Descriptive statistics and correlations between the six subscales of the SCS, the ICG total score, and NEO-FFI-3 neuroticism are presented in Table 1. As shown in Table 1, the total score of the ICG was significantly positively correlated 
with the negative dimensions of SC and the negative SC composite. Correlations ranged from .30 (self-judgment) to .37 (isolation and self-coldness composite). Further, the ICG total score was significantly positively correlated with NEO-FFI-3 neuroticism. Bivariate correlations between the SCS scales and NEO-FFI-3 neuroticism are also displayed in table 1 showing high positive correlation coefficients for the negative SCS scales (ranging from .64 for self-judgment to .76 for the self-coldness composite) and medium-sized negative correlations for the positive SCS scales (ranging from -.27 for common humanity to -.43 for self-kindness). Results from regression analysis predicting the ICG total score from SCS dimension scales controlled for sample characteristics are presented in table 2. Results showed that the SCS dimension scales predicted significantly the ICG total score beyond the control variables and explained additional $11 \%$ of the variance. The self-kindness and isolation subscales of the SCS emerged as significant positive individual predictors $(p<.05)$. However, as the correlation analysis showed a weak and nonsignificant bivariate association of self-kindness with the ICG total score, the positive relationship in the regression analysis is likely due to a suppressor effect.

Prior to analyses testing for moderation of the self-kindness composite between cause of death and neuroticism and symptoms of CG, the associations between cause of death and the six subscales of the SCS were examined using a series of one-way ANOVAs. Because of the small number of participants bereaved by suicide in the sample, this group was included the group of participants who were bereaved by sudden and unnatural deaths. Results from the analyses are shown in Table 3 and reveal that there was a main effect of group on the mindfulness scale of the SCS, but post hoc group comparisons were not significant at $p<.05$.

Results from the two regression analyses testing moderation of self-kindness between the risk factors neuroticism and cause of death and complicated grief symptoms are displayed in 
Table 4. The regression models with cause of death $(F=3,95,5,146, p=.002)$ and neuroticism $(F=12,93,3,148, p<.001)$ as predictors were significant and explained $12 \%$ and $21 \%$ of the variance of the ICG scores, respectively. However, the interaction term of the two predictors and the self-kindness composite as moderator were nonsignificant in both regression models.

\section{Discussion}

The purpose of this study was to explore the relationships between $\mathrm{SC}$ and the severity of the grief reaction (as measured by the ICG) in individuals who are bereaved by the loss of a loved one. Based on previous findings suggesting that SC buffers against the development of mental health problems (e.g., Körner et al., 2015; Marshall et al., 2015), it was further examined if self-kindness moderates the relationships between established risk factors for CG (i.e., cause of death and neuroticism) and symptoms of CG after bereavement.

Results from correlation and regression analyses showed significant associations between the negative dimensions of SC (i.e., self-judgment, isolation, and over-identification) and CG symptomatology. Thus, being critical and harsh towards oneself is related to experiencing more severe grief reactions after a loss. This finding is consistent with the cognitive-behavioral formulation of CG (Boelen et al., 2006) and the results of previous investigations demonstrating that negative cognitions about the self account for a substantial proportion of the variance in grief symptoms (e.g., Boelen, van den Bout, \& van den Hout, 2003). Further, over-identifying with emotions instead of observing them with a non-judgmental and accepting attitude is associated with higher levels of CG symptoms. Over-identification with negative emotions involves being caught and fixated on these feelings and to react to them in an automatic and habitual way (Bishop et al., 2004). However, when controlling for death related variables and the 
other dimensions of SC, only the isolation subscale of the SCS emerged as a significant individual predictor of $\mathrm{CG}$. In the context of $\mathrm{SC}$, isolation refers to feeling alone and separated when confronted with failure and inadequacy as opposed to being aware of that one's suffering is part of being human and connecting oneself to humanity (Neff \& Dahm, 2015). This finding aligns with previous studies showing strong connections between bereavement, loneliness, and mental health problems (e.g., Fried et al., 2015). Further, consistent with previous findings (Neff et al., 2007; Pfattheicher et al., 2017), significant associations between SC and neuroticism were found also in this sample of bereaved individuals with the negative dimensions of SC showing higher correlations with neuroticism than the positive dimensions. Finally, replicating previous findings (Burke \& Neimeyer, 2013), significant associations between neuroticism and cause of death with symptoms of CG were found. However, self-kindness was not found to moderate these relationships. Taken together, significant correlations with the negative dimensions of CG and the lack of associations with the positive dimensions of CG suggest that low $\mathrm{SC}$ is a vulnerability factor for CG. Self-kindness does not seem to buffer the effects of neuroticism and unexpected and unnatural death on the severity of grief symptoms.

A shortcoming of the present study is that the measurements were performed in a nonclinical sample with an overweight of female respondents. The majority of participants (78\%) did not show scores above the clinical threshold for CG on the ICG. Similarly, participants grieving the loss of a child or by suicide are weakly represented in the current sample. Several studies have shown that women and men show a different pattern in grief reactions, where women are often more emotionally focused, while men more often concentrate on practical reorientation in life after the loss (Stroebe, Stroebe, \& Schut, 2001). Bereavement by suicide has been particularly linked to feelings of guilt (Jordan, 2001). Thus, the composition of the sample 
with regard to the level of CG symptomatology, gender, relationship with the deceased, and cause of death may have affected the results obtained in the current investigation and may limit the generalizability of findings. Importantly, the cross-sectional design of the study allows no conclusions about the causal directions of the observed relationships. Thus, based on the current findings, it cannot be determined whether low SC causes CG symptoms or CG symptoms cause low SC or if there is a reciprocal relationship between these two constructs. Longitudinal studies are needed to clarify possible causal mechanisms that underlie these relationships. The limitations of the present investigation with respect to sample characteristics and the crosssectional nature of the analyses prevent treatment recommendations to clinicians at this stage of research as the importance of SC as a treatment target is not clear.

Despite these limitations, the current findings suggest that clinicians should be aware of the associations between self-criticalness, experiencing one's own suffering as isolating from the social world, and over-identification with emotions and the severity of grief responses in bereaved individuals. Clinicians should be attentive to how the three components of low selfcompassion may affect the grief process over time.

In conclusion, the results of the present study suggest a relationship between low SC and the severity of grief reactions after the loss of a loved one. Future studies should elucidate these relationships further using clinical samples and repeated measurements. 


\section{References}

Barlow, D. H., Sauer-Zavala, S., Carl, J. R., Bullis, J. R., \& Ellard, K. K. (2014). The nature, diagnosis, and treatment of neuroticism: Back to the future. Clinical Psychological Science, 2, 344-365. doi: 10.1177/2167702613505532

Bishop, S. R., Lau, M., Shapiro, S., Carlson, L., Anderson, N. D., Carmody, J., . . Devins, G. (2004). Mindfulness: A proposed operational definition. Clinical Psychology: Science and Practice, 11, 230-241. doi: 10.1093/clipsy.bph077

Boelen, P. A., van den Bout, J., \& van den Hout, M. A. (2003). The role of cognitive variables in psychological functioning after the death of a first degree relative. Behaviour Research and Therapy, 41, 1123-1136. doi: 10.1016/s0005-7967(02)00259-0

Boelen, P. A., van den Hout, M. A., \& van den Bout, J. (2006). A cognitive-behavioral conceptualization of complicated grief. Clinical Psychology: Science and Practice, 13, 109-128. doi: 10.1111/j.1468-2850.2006.00013.x

Bonanno, G. A., Wortman, C. B., Lehman, D. R., Tweed, R. G., Haring, M., Sonnega, J., . . Nesse, R. M. (2002). Resilience to loss and chronic grief: A prospective study from preloss to 18-months postloss. Journal of Personality and Social Psychology, 83, 11501164. doi: $10.1037 / 0022-3514.83 .5 .1150$

Braun, T. D., Park, C. L., \& Gorin, A. (2016). Self-compassion, body image, and disordered eating: A review of the literature. Body Image, 17, 117-131. doi: 10.1016/j.bodyim.2016.03.003

Brenner, R. E., Heath, P. J., Vogel, D. L., \& Credé, M. (2017). Two is more valid than one: Examining the factor structure of the Self-Compassion Scale (SCS). Journal of Counseling Psychology, 64, 696-707. doi: 10.1037/cou0000211 
Burke, L. A., \& Neimeyer, R. A. (2013). Prospective risk factors for complicated grief: A review of the empirical literature. In M. Stroebe, H. Schut, \& J. Van den Bout (Eds.), Complicated grief: Scientific foundations for health care professionals (pp. 145-161). East Sussex: Routledge.

Costa, J., Marôco, J., Pinto-Gouveia, J., Ferreira, C., \& Castilho, P. (2016). Validation of the psychometric properties of the Self-Compassion Scale. Testing the factorial validity and factorial invariance of the measure among borderline personality disorder, anxiety disorder, eating disorder and general populations. Clinical Psychology \& Psychotherapy, 23, 460-468. doi: 10.1002/cpp.1974

Dundas, I., Svendsen, J. L., Wiker, A. S., Granli, K. V., \& Schanche, E. (2016). Self-compassion and depressive symptoms in a Norwegian student sample. Nordic Psychology, 68, 58-72. doi: $10.1080 / 19012276.2015 .1071203$

Dyregrov, A. (2006). Komplisert sorg: Teori og behandling. Tidsskrift for Norsk Psykologforening, 43, 779-786.

Eicher, A. C., Davis, L. W., \& Lysaker, P. H. (2013). Self-compassion: A novel link with symptoms in schizophrenia? Journal of Nervous and Mental Disease, 201, 389-393. doi: 10.1097/NMD.0b013e31828e10fa

Fried, E. I., Bockting, C., Arjadi, R., Borsboom, D., Amshoff, M., Cramer, A. O., . . Stroebe, M. (2015). From loss to loneliness: The relationship between bereavement and depressive symptoms. Journal of Abnormal Psychology, 124, 256-265. doi: 10.1037/abn0000028

Gilbert, P. (2009). Introducing compassion-focused therapy. Advances in Psychiatric Treatment, 15, 199-208. doi: 10.1192/apt.bp.107.005264 
Hayes, A. F. (2017). Introduction to mediation, moderation, and conditional process analysis: A regression-based approach (2nd ed.). New York: Guilford.

Jordan, J. R. (2001). Is suicide bereavement different? A reassessment of the literature. Suicide and Life-Threatening Behavior, 31,91-102. doi: 10.1521/suli.31.1.91.21310

Kaltman, S., \& Bonanno, G. A. (2003). Trauma and bereavement:: Examining the impact of sudden and violent deaths. Journal of Anxiety Disorders, 17, 131-147. doi: $10.1016 / \mathrm{S} 0887-6185(02) 00184-6$

Kristensen, P., Weisæth, L., \& Heir, T. (2012). Bereavement and mental health after sudden and violent losses: A review. Psychiatry: Interpersonal and Biological Processes, 75, 76-97. doi: $10.1521 /$ psyc.2012.75.1.76

Kristensen, P., Weisæth, L., Hussain, A., \& Heir, T. (2015). Prevalence of psychiatric disorders and functional impairment after loss of a family member: A longitudinal study after the 2004 tsunami. Depression and Anxiety, 32, 49-56. doi: 10.1002/da.22269

Kübler-Ross, E. (2009). On death and dying: Taylor \& Francis.

Körner, A., Coroiu, A., Copeland, L., Gomez-Garibello, C., Albani, C., Zenger, M., \& Brähler, E. (2015). The role of self-compassion in buffering symptoms of depression in the general population. PloS one, 10, e 0136598 .

López, A., Sanderman, R., Smink, A., Zhang, Y., van Sonderen, E., Ranchor, A., \& Schroevers, M. J. (2015). A reconsideration of the Self-Compassion Scale's total score: Selfcompassion versus self-criticism. PloS one, 10, e0132940.

Lundorff, M., Holmgren, H., Zachariae, R., Farver-Vestergaard, I., \& O’Connor, M. (2017). Prevalence of prolonged grief disorder in adult bereavement: A systematic review and 
meta-analysis. Journal of Affective Disorders, 212, 138-149. doi:

10.1016/j.jad.2017.01.030

MacBeth, A., \& Gumley, A. (2012). Exploring compassion: A meta-analysis of the association between self-compassion and psychopathology. Clinical Psychology Review, 32, 545552. doi: 10.1016/j.cpr.2012.06.003

Marshall, S. L., Parker, P. D., Ciarrochi, J., Sahdra, B., Jackson, C. J., \& Heaven, P. C. L. (2015). Self-compassion protects against the negative effects of low self-esteem: A longitudinal study in a large adolescent sample. Personality and Individual Differences, 74, 116-121. doi: 10.1016/j.paid.2014.09.013

McCrae, R. R., \& Costa, P. T. (2010). NEO inventories for the NEO Personality Inventory-3 (NEO PI-3), Neo Five-Factor Inventory-3 (NEO FFI-3) and NEO Personality InventoryRevised (NEO PI-R): Professional manual. Lutz, Fl: Psychological Assessment Resources.

McNeish, D. (2018). Thanks coefficient alpha, we'll take it from here. Psychological Methods, 23, 412-433. doi:10.1037/met0000144

Muris, P., Otgaar, H., \& Petrocchi, N. (2016). Protection as the mirror image of psychopathology: Further critical notes on the self-compassion scale. Mindfulness, 7, 787-790. doi: 10.1007/s12671-016-0509-9

Muris, P., \& Petrocchi, N. (in press). Protection or vulnerability? A meta-analysis of the relations between the positive and negative components of self-compassion and psychopathology. Clinical Psychology \& Psychotherapy. doi: 10.1002/cpp.2005

Neff, K. (2003a). The development and validation of a scale to measure self-compassion. Self and Identity, 2, 223-250. doi: 10.1080/15298860309027 
Neff, K. (2003b). Self-compassion: An alternative conceptualization of a healthy attitude toward oneself. Self and Identity, 2, 85-101. doi: 10.1080/15298860309032

Neff, K. D. (2016). The self-compassion scale is a valid and theoretically coherent measure of self-compassion. Mindfulness, 7, 264-274. doi: 10.1007/s12671-015-0479-3

Neff, K. D., \& Dahm, K. A. (2015). Self-compassion: What it is, what it does, and how it relates to mindfulness. In B. D. Ostafin, M. D. Robinson, \& B. P. Meier (Eds.), Mindfulness and self-regulation (pp. 121-137). New York: Springer.

Neff, K. D., Rude, S. S., \& Kirkpatrick, K. (2007). An examination of self-compassion in relation to positive psychological functioning and personality traits. Journal of Research in Personality, 41, 908-916. doi: 10.1016/j.jrp.2006.08.002

Neff, K. D., \& Vonk, R. (2009). Self-compassion versus global self-esteem: Two different ways of relating to oneself. Journal of Personality, 77, 23-50. doi: 10.1111/j.14676494.2008.00537.x

Neff, K. D., Whittaker, T. A., \& Karl, A. (2017). Examining the factor structure of the selfcompassion scale in four distinct populations: Is the use of a total scale score justified? Journal of Personality Assessment, 99, 596-607. doi: 10.1080/00223891.2016.1269334

Peters, G.-J. Y. (2014). The alpha and the omega of scale reliability and validity: Why and how to abandon Cronbach's alpha and the route towards more comprehensive assessment of scale quality. European Health Psychologist, 16, 56-69.

Pfattheicher, S., Geiger, M., Hartung, J., Weiss, S., \& Schindler, S. (2017). Old wine in new bottles? The case of self-compassion and neuroticism. European Journal of Personality, 31, 160-169. doi: 10.1002/per.2097 
Piper, W. E., Ogrodniczuk, J. S., Joyce, A. S., \& Weideman, R. (2011). Short-term group therapies for complicated grief: Two research-based models. Washington, DC: American Psychological Association.

Prigerson, H. G., Horowitz, M. J., Jacobs, S. C., Parkes, C. M., Aslan, M., Goodkin, K., . . . Maciejewski, P. K. (2009). Prolonged grief disorder: Psychometric validation of criteria proposed for DSM-V and ICD-11. PLoS Med, 6, e1000121. doi: 10.1371/journal.pmed.1000121

Prigerson, H. G., Maciejewski, P. K., Reynolds Iii, C. F., Bierhals, A. J., Newsom, J. T., Fasiczka, A., . . Miller, M. (1995). Inventory of Complicated Grief: A scale to measure maladaptive symptoms of loss. Psychiatry Research, 59, 65-79. doi: 10.1016/0165$1781(95) 02757-2$

Revelle, W. (2017). psych: Procedures for Personality and Psychological Research. https://CRAN.R-project.org/package=psych Version $=1.7 .5$.

Rude, S. S., Little Maestas, K., \& Neff, K. (2007). Paying attention to distress: What's wrong with rumination? Cognition and Emotion, 21, 843-864. doi: $10.1080 / 02699930601056732$

Shapiro, S. L., \& Carlson, L. E. (2009). The art and science of mindfulness. Washington, DC: American Psychological Association.

Shear, M. K. (2012). Grief and mourning gone awry: Pathway and course of complicated grief. Dialogues in clinical neuroscience, 14, 119-128.

Shear, M. K. (2014). How To: Cultivate Self-Compassion. Retrieved from http://modernloss.com/self-compassion/ 
Shear, M. K., Ghesquiere, A., \& Glickman, K. (2013). Bereavement and complicated grief. Current Psychiatry Reports, 15:406. doi: 10.1007/s11920-013-0406-z

Shear, M. K., Simon, N., Wall, M., Zisook, S., Neimeyer, R., Duan, N., . . Keshaviah, A. (2011). Complicated grief and related bereavement issues for DSM-5. Depression and Anxiety, 28, 103-117. doi: 10.1002/da.20780

Stroebe, M., \& Schut, H. (1999). The dual process model of coping with bereavement: Rationale and description. Death Studies, 23, 197-224. doi: 10.1080/074811899201046

Stroebe, M., \& Schut, H. (2010). The dual process model of coping with bereavement: A decade on. Omega-Journal of Death and Dying, 61, 273-289. doi: 10.2190/OM.61.4.b

Stroebe, M., Schut, H., \& Stroebe, W. (2007). Health outcomes of bereavement. Lancet, 370, 1960-1973. doi: 10.1016/s0140-6736(07)61816-9

Stroebe, M., Stroebe, W., \& Schut, H. (2001). Gender differences in adjustment to bereavement: An empirical and theoretical review. Review of General Psychology, 5, 62-83. doi: $10.1037 / 1089-2680.5 .1 .62$

Wijngaards-de Meij, L., Stroebe, M., Schut, H., Stroebe, W., van den Bout, J., van der Heijden, P., \& Dijkstra, I. (2007). Neuroticism and attachment insecurity as predictors of bereavement outcome. Journal of Research in Personality, 41, 498-505. doi: 10.1016/j.jrp.2006.06.001

Wortman, C. B., \& Boerner, K. (2011). Beyond the myths of coping with loss: Prevailing assumptions versus scientific evidence. In H. S. Friedman (Ed.), The Oxford Handbook of Health Psychology (p. 438-476). New York: Oxford University Press. 